\title{
Biological Action Identification Does Not Require Early Visual Input for Development
}

\author{
Siddhart S. Rajendran, ${ }^{1,2}$ Davide Bottari, ${ }^{3}$-Idris Shareef, ${ }^{1,2}$ Kabilan Pitchaimuthu, ${ }^{1}{ }^{\circ}$ Suddha Sourav, ${ }^{1}$ \\ Nikolaus F. Troje, ${ }^{4}$ Ramesh Kekunnaya, ${ }^{2}$ and Brigitte Röder ${ }^{1}$
}

https://doi.org/10.1523/ENEURO.0534-19.2020

${ }^{1}$ Biological Psychology and Neuropsychology, University of Hamburg, Hamburg, Germany, ${ }^{2}$ Jasti V Ramanamma Children's Eye Care Center, LV Prasad Eye Institute, Hyderabad, India, ${ }^{3}$ IMT School for Advanced Studies Lucca, Lucca, Italy, and ${ }^{4}$ Department of Biology, Centre for Vision Research, York University, Toronto, Ontario, Canada

\begin{abstract}
Visual input during the first years of life is vital for the development of numerous visual functions. While normal development of global motion perception seems to require visual input during an early sensitive period, the detection of biological motion (BM) does not seem to do so. A more complex form of BM processing is the identification of human actions. Here, we tested whether identification rather than detection of BM is experience dependent. A group of human participants who had been treated for congenital cataracts (CC; of up to 18 years in duration, CC group) had to identify ten actions performed by human line figures. In addition, they performed a coherent motion (CM) detection task, which required identifying the direction of $\mathrm{CM}$ amid the movement of random dots. As controls, developmental cataract (DC) reversal individuals (DC group) who had undergone the same surgical treatment as CC group were included. Moreover, normally sighted controls were tested both with vision blurred to match the visual acuity (VA) of CC individuals [vision matched (VM) group] and with full sight [sighted control (SC) group]. The CC group identified biological actions with an extraordinary high accuracy (on average $\sim 85 \%$ correct) and was indistinguishable from the VM control group. By contrast, CM processing impairments of the CC group persisted even after controlling for VA. These results in the same individuals demonstrate an impressive resilience of BM processing to aberrant early visual experience and at the same time a sensitive period for the development of CM processing.
\end{abstract}

Key words: biological action; biological motion; congenital cataract; global motion; visual deprivation

\section{Significance Statement}

Biological motion (BM) is a crucial aspect of human vision, which has been shown to emerge early in human ontogeny. Here, we report an astonishing high accuracy in identifying human actions in a unique group of individuals who had regained vision later in life (until the age of 18 years) after being treated for congenital cataracts (CC). By contrast the same individuals were markedly impaired in another non-BM tasks requiring the detection of motion coherence in dot kinematograms, even after visual acuity (VA) impairments were taken into account. Thus, the present study demonstrates a remarkable resilience of complex BM processing capabilities such as the identification of human actions to aberrant early visual experience.

\section{Introduction}

Sensory input during early years of life is essential for normal development of sensory systems (Wiesel and Hubel, 1965). In humans, studies in individuals treated for

Received December 16, 2019; accepted August 27, 2020; First published October 15, 2020.

The authors declare no competing financial interests. congenital cataracts (CC) have revealed incomplete recovery in many visual functions, including visual acuity (VA; Ellemberg et al., 1999), stereovision (Tytla et al., 1993), visual feature binding (Putzar et al., 2007; McKyton

Author contributions: S.S.R., D.B., S.S., N.F.T., and B.R. designed research; S.S.R., D.B., I.S., K.P., S.S., R.K., and B.R. performed research; S.S.R. analyzed data; S.S.R. wrote the paper. 
et al., 2015), global motion processing (Ellemberg et al., 2002; Bottari et al., 2018), and face processing (Le Grand et al., 2001; Röder et al., 2013), while functions such as color discrimination (Brenner et al., 1990; Pitchaimuthu et al., 2019) seemed to emerge independently of early visual experience. Biological motion (BM) processing, e.g., the ability to detect the movement of biological figures with sparse information (Johansson, 1973), has been shown to recover well following early visual deprivation (Hadad et al., 2012), that is, both detection thresholds as well as neural signatures have been observed to be indistinguishable between a CC group and normally sighted controls (SC group; Bottari et al., 2015, 2016).

However, it is unclear yet whether more complex aspects of BM processing, such as the identification of human actions, require early visual experience. BM detection, as studied with point light displays of walkers, requires the extraction of the spatial configuration of point lights and its congruent change over time (Theusner et al., 2014). In contrast, action identification involves knowledge of the meaning of human postures and how they change over time. Action identification is further complicated by the large variance from different agents performing the actions and the different viewpoints from which actions are observed. Action identification, thus, requires extracting action invariant features.

In the present study, we tested individuals with a history of pattern vision loss because of bilateral, total, dense CC on their ability to accurately perceive human actions performed by line figures. For this purpose, we used the test battery (BMLtest battery) developed by Saunders and Troje (2011). Three potential confounds were additionally investigated: first, unspecific effects related to cataract surgery; second, the timing of visual deprivation, that is, whether the visual deprivation existed at birth or emerged later in development; third, the overall lower VA typical for individuals with a history of early cataracts. In previous studies, both of these confounds have been simultaneously controlled for by including individuals who had lower VA compared with typically sighted individuals because of cataract onset later during development [e.g., individuals with reversed developmental cataract (DC); Lewis and Maurer, 2009; Röder et al., 2013]. Alternatively, the persisting visual impairments have been controlled for by blurring visual stimuli for normally SCs (McKyton et al., 2015). If a sensitive period exists for the development of a specific function, once VA is controlled for, the CC group,

The work was supported by the European Research Council Grant ERC-2009AdG 249425-CriticalBrainChanges and the Deutsche Forschungsgemeinschaft Grant Ro 2625/10-1 (to B.R.).

Acknowledgements: We thank Prof. Balasubramanian for supporting the study at the Hyderabad Eye Research Foundation and the Opticals department at LV Prasad Eye Institute for preparing the trial frames with the Bangerter filters.

Correspondence should be addressed to Siddhart S. Rajendran at siddhart. srivatsav.rajendran@uni-hamburg.de.

https://doi.org/10.1523/ENEURO.0534-19.2020

Copyright @ 2020 Rajendran et al.

This is an open-access article distributed under the terms of the Creative Commons Attribution 4.0 International license, which permits unrestricted use, distribution and reproduction in any medium provided that the original work is properly attributed. but not the DC group is expected to show impaired performance. The normally sighted age matched controls were tested with full sight and with the VA matched using specialized translucent filters called Bangerter filters, which have been regularly used in amblyopia therapy (Agervi, 2011). All participants were tested in two tasks of the BMLtest battery (Saunders and Troje, 2011): (1) an action identification task, in which subjects had to name the action performed by an actor shown as stick figures; (2) a coherent motion (CM) detection task, in which participants judged the direction of the CM of dots among a set of randomly moving dots (CM task).

We predicted that complex aspects of BM, such as the identification of human actions, would depend less on early visual experience than CM perception. Thus, we hypothesized that any group difference between the CC group and SC group in the action identification task can be accounted for by VA differences. By contrast, for the $\mathrm{CM}$ task we predicted that impairments specific for the same CC group emerge even after controlling for VA. Thus, the present study is aimed at providing non-confounded evidence for a sensitive phase in early development for global motion processing while showing in the same individuals a high resilience of complex BM functions as the identification of human actions to aberrant early visual experience.

\section{Materials and Methods}

\section{Participants}

The group of individuals with a transient phase of CC (CC group) was comprised of 12 participants (mean age: 16 years, range: 7-34 years; three females; all right handed; mean age at surgery: 66.8 months, range: 2220 months; mean duration since surgery: 120 months, range: 6-396 months; for detailed demographics of participants, refer to Table 1) who had undergone treatment for bilateral, dense, CC in both eyes. These participants were recruited from LV Prasad Eye Institute, Hyderabad, India. Congenital cataract individuals qualifying for the present study were identified among a larger number of patients with the diagnosis "congenital cataract" based on the following criteria. (1) All patients suffered nystagmus which is often the result of a lack of pattern vision for longer than 10-13 weeks after birth (Rogers et al., 1981; Gelbart et al., 1982). Such a nystagmus persists after surgery, and was present in all CC individuals. (2) The lenticular opacity for CC individuals was dense before surgery which resulted in an inability to have a view of the fundus during clinical examination (except participants with partially absorbed cataracts). Additionally, (3) a positive family history of congenital cataract. (4) Esotropia resulting from equal visual deprivation in both eyes was used as additional classification criteria. This information was available from the detailed medical records available at LV Prasad Eye Institute, Hyderabad, India. Patients with an ambiguous clinical profile were not included. Although a $100 \%$ confidence of having included only congenital cataract-reversal individuals with a history of bilateral, total, dense cataracts is not possible in a retrospective 
Table 1: Clinical information of the congenital cataract reversal group (CC group) and age/sex matching with the VM control group

\begin{tabular}{|c|c|c|c|c|c|c|c|c|c|c|c|c|c|}
\hline Participant & Strabismus & $\begin{array}{l}\text { Family } \\
\text { history }\end{array}$ & $\begin{array}{l}\text { VA at surgery } \\
\text { (better eye) }\end{array}$ & Dense & $\begin{array}{l}\text { Nystag- } \\
\text { mus }\end{array}$ & $\begin{array}{l}\text { Age at } \\
\text { surgery } \\
\text { (months) }\end{array}$ & $\begin{array}{l}\text { Duration } \\
\text { since } \\
\text { surgery } \\
\text { (months) }\end{array}$ & $\begin{array}{l}\text { Age } \\
\text { at test } \\
\text { (years) }\end{array}$ & $\begin{array}{l}\text { VA at test } \\
\text { (logMAR) }\end{array}$ & $\begin{array}{l}\text { Sex } \\
\#\end{array}$ & $\begin{array}{l}\text { Age } \\
\text { VM } \\
\text { (years) }\end{array}$ & $\begin{array}{l}\text { VA } \\
\text { VM } \\
(\log M A R)\end{array}$ & $\begin{array}{l}\text { Sex } \\
\text { VM } \\
\#\end{array}$ \\
\hline $\mathrm{CC} 2$ & Exotropia & Yes & $\begin{array}{l}\text { Fixates and follows } \\
\text { light }\end{array}$ & Yes & Yes & 5 & 139 & 12 & 0.48 & M & 12 & 0.5 & M \\
\hline CC3 & Esotropia & Yes & $\begin{array}{l}\text { Fixates and follows } \\
\text { light }\end{array}$ & Yes & Yes & 2 & 106 & 9 & 0.3 & $\mathrm{~F}$ & 9 & 0.39 & $\mathrm{~F}$ \\
\hline CC5 & Esotropia & Yes & NA & Yes & Yes & 24 & 396 & 34 & 0.3 & $M$ & 32 & 0.37 & $\mathrm{~F}$ \\
\hline CC6 & No & Yes & NA & Yes & Yes & 72 & 348 & 30 & 1.3 & M & 29 & 1.38 & $\mathrm{~F}$ \\
\hline $\mathrm{CC} 7$ & Esotropia & No & PL/PR accurate & Yes & Yes & 121 & 11 & 11 & 0.8 & $\mathrm{~F}$ & 11 & 0.85 & $\mathrm{~F}$ \\
\hline $\mathrm{CC} 8$ & No & Yes & $\mathrm{HM}+$ & Yes & Yes & 86 & 34 & 10 & 0.5 & M & 11 & 0.55 & M \\
\hline CC9 & No & No & PL/PR accurate & Yes & Yes & 220 & 6 & 18 & 1.08 & M & 19 & 0.9 & $\mathrm{~F}$ \\
\hline
\end{tabular}

*Subject with partially absorbed cataract; abbreviations used in VA columns: NA, not available in records; PL, perception of light; PR, perception of the direction of light rays; HM, hand movement close to face; \#: M, male; F, female.

classification of participants, the likelihood of achieving an accurate CC classification was maximized in the present sample by having access to extensive clinical data allowing for the use of strict criteria.

In order to ensure that any observed difference between congenital cataract-reversal individuals and normally SCs was not the result of trivial causes such as the experience of a surgical procedure at the eyes, and to understand the effects of vision loss immediately after birth versus later in childhood, we tested a group of control participants whose form vision was preserved at birth and who developed cataracts later during childhood. These participants had undergone the same surgical procedure as the CC group; three out of these 12 individuals had congenital but non-dense cataracts. These participants were subsumed as DC group. Similar to the CC group, these participants had undergone treatment for cataract (mean e at surgery: 116 months, range: 24-434 months; mean time since surgery: 37 months, range: 2-183 months). We tested 12 DC participants (mean age: 12.6 years, range: 8-37 years, four females, all right-handed) who were recruited from LV Prasad Eye Institute, Hyderabad, India. They were classified based on the medical records of LV Prasad Eye Institute, India. The clinical diagnosis of DC was mostly based on a number of criteria such as the parents' or patients' report of the age of poor vision onset, lack of nystagmus and a lack of family history of congenital eye pathologies. Additionally, individuals with incomplete congenital cataract, suggesting preservation of early pattern vision were included in the DC group (for complete description, see Table 2).

Table 2: Clinical information of the DC group

\begin{tabular}{|c|c|c|c|c|c|c|c|c|c|c|}
\hline Participant & Strabismus & $\begin{array}{l}\text { Family } \\
\text { history }\end{array}$ & $\begin{array}{l}\text { VA at surgery } \\
\text { (better eye) }\end{array}$ & Dense & $\begin{array}{l}\text { Nystag- } \\
\text { mus }\end{array}$ & $\begin{array}{l}\text { Age at } \\
\text { surgery } \\
\text { (months) }\end{array}$ & $\begin{array}{l}\text { Duration } \\
\text { since } \\
\text { surgery } \\
\text { (months) }\end{array}$ & $\begin{array}{l}\text { Age } \\
\text { at test } \\
\text { (years) }\end{array}$ & $\begin{array}{l}\text { VA at test } \\
\text { (logMAR) }\end{array}$ & $\begin{array}{l}\text { Sex } \\
\#\end{array}$ \\
\hline DC2 & NA & NA & NA & NA & NA & 102 & 43 & 12 & 0.18 & M \\
\hline DC3 & Exotropia & No & fixates and follows objects & No & Yes & 24 & 183 & 17 & 0.18 & $\mathrm{~F}$ \\
\hline DC4 & NA & NA & NA & NA & NA & 120 & 20 & 11 & 0.18 & $\mathrm{~F}$ \\
\hline $\mathrm{DC} 7^{\S}$ & NA & Yes & $20 / 200$ & No & Yes & 434 & 9 & 36 & 1 & M \\
\hline DC8 & No & No & $\mathrm{PL}+\mathrm{PR}$ accurate & Yes & Yes & 110 & 10 & 10 & 1.7 & M \\
\hline DC9 & No & No & $20 / 100$ & No & No & 97 & 6 & 8 & 0 & M \\
\hline DC10 & NA & No & $20 / 80$ & No & No & 84 & 11 & 7 & 0.3 & $M$ \\
\hline DC11 & Exotropia & No & CF $2 \mathrm{~m}$ & No & No & 96 & 25 & 10 & 0 & $\mathrm{~F}$ \\
\hline DC12 & Exotropia & No & CF $1 \mathrm{~m}$ & No & Yes & 105 & 2 & 8 & 1.3 & $M$ \\
\hline
\end{tabular}

\footnotetext{
$\S$ Individuals with incomplete congenital cataract; abbreviations used in VA columns: NA, not available in records; PL, perception of light; PR, perception of the di-
} rection of light rays; $\mathrm{HM}$, hand movement close to face; \#: $\mathrm{M}$, male; $\mathrm{F}$, female. 
To control for VA and thus to isolate the effects of prevailing VA loss at the time of testing, a second control group of 12 participants with normal vision (mean age 15.3 years, range: 8-32 years; 6 females; all right-handed; for the exact VAs, refer to Table 1) was tested. Similar to the CC and DC participants, these individuals were also recruited from Hyderabad, India. They were matched for age with the $\mathrm{CC}$ group (CC; \pm 1 year, for one adult $\mathrm{CC}$ participant, 2 years, see Table 1). This control group performed the task twice: once with normal vision and the second time with the VA being individually matched to one of the $\mathrm{CC}$ individuals. With their vision reduced using the Bangerter filters, these participants served as the vision matched (VM) control group, and without the filters the same participants served as normally SCs. Hence, with their reduced vision, the control group was matched for age and VA with that of the CC group.

Participants in all three groups (CC, DC, VM/SC) were healthy (expect the history of cataracts in CC and DC individuals) with no history of physical problems by self-report (VM/SC group) and by physical examination (CC and DC groups). The study was approved by the institutional ethics boards of University of Hamburg, Hamburg, Germany and LV Prasad Eye Institute, Hyderabad, India. Informed consent was obtained from participants, and from legal guardians for minors before the beginning of experiments. The quality of health care received by the participants was not affected by their willingness to participate in our experiments.

\section{Stimuli and apparatus}

\section{VA matching using Bangerter filters}

Bangerter filters are translucent light diffusers that can be attached to spectacles and are capable of causing degradation in vision because of the resultant attenuation of higher spatial frequencies (Pérez et al., 2010). Although these filters can reduce the VA in steps on 0.1 logMAR in healthy eyes (Odell et al., 2008; Agervi, 2011; Rutstein et al., 2011), an intended degradation of $>1.0$ logMAR of VA cannot be obtained even by the manufacturer's recommendation. Since our participants required VA reduction of up to 1.3 logMAR, we used a combination of filters to achieve VA reductions beyond $1.0 \log M A R$. For this purpose, we attached the filters to zero power wide field trial lenses typically used in optometric eye examinations. Similar to the use of trial lenses during an eye examination to arrive at the correct refractive correction, combinations of these "trial filters" were in turn used to arrive at the desired VA. Using the manufacturer's recommendations and previous reports (Odell et al., 2008) as starting filter strengths, different combinations of filters were tried until the desired VA was obtained. Although not tested with Bangerter filters, the neural system is generally prone to blur adaptation (Clifford et al., 2007; Webster, 2015), and hence, the participants were asked to stay with the filters for a few minutes for the visual system to get adapted to the blur, and VA estimation was repeated after adaptation. If the pre and post VA varied widely, the filter strength was assessed again until a stable filter strength was obtained. While combining multiple filters, the "microbubbles"
(Pérez et al., 2010) of a given filter aligning with that of the preceding/following one might potentially be an important factor in maintaining image degradation. But in our experience, we noticed that the alignment was robust to small variations in head movements. All VA measurements were done using the Landodt's $\mathrm{C}$ optotype of the Freiburg Visual Acuity Test (FrACT; Bach, 1996). The optotypes were shown on a 20" Dell monitor with a resolution of $1600 \times 900$ (refresh rate $60 \mathrm{~Hz}$ ) and at a testing distance of $240 \mathrm{~cm}$ controlled by a Dell laptop.

\section{Stimuli}

The biomotion test battery (BMLtest) by Saunders and Troje (2011) was used. The test battery consists of a number of tests, of which we used the "coherence" and "action" experiments. All stimuli were shown on a 20" Dell IN2030M LCD monitor with a resolution of $1600 \times 900$ and a refresh rate of $60 \mathrm{~Hz}$ using a Dell laptop. Participants were seated at a distance of $60 \mathrm{~cm}$ from the screen, and viewed the stimuli with their best refractive correction. They were allowed to switch between the segments of their bifocals (in case of the CC and DC participants) and were additionally allowed to get a closer look at the stimuli in cases where the stimuli were not sufficiently visible at the testing distance. For the VM participants the Bangerter filters were placed over their refractive correction.

\section{Procedure \\ CM task}

The "coherency test" of BMLtest battery, originally based on Newsome and Paré (1988), and used by Bottari et al. (2018) was adapted to obtain global motion coherence thresholds (CM task). In a $10^{\circ}$ circular field, 15 dots, each of 15 pixel size moved either randomly or were displaced by $0.15^{\circ}$ in a particular direction (right or left; the direction of motion was randomized on each trial). Each trial lasted $1000 \mathrm{~ms}$ and was preceded by a black screen with white fixation cross for the same amount of time. After each trial, participants gave a verbal response indicating the direction of motion (toward left or toward right). Younger children waved the appropriate hand to indicate the direction instead of providing an oral response. In all cases, the experimenter pressed the response button. The next trial did not start until participants had given a response for the current trial. The test used a QUEST staircase procedure to obtain the threshold for detecting the direction of motion with a likelihood of $82 \%$.

\section{Biological action identification task}

The action subtest of the BMLtest battery was used to assess participants' ability to accurately recognize an action performed by a moving line figure (see Fig. 1). The suite consisted of 10 different actions presented from three different viewpoints: straight ahead $\left(0^{\circ}\right.$ view angle),

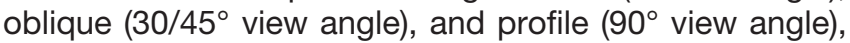
totaling to 30 trials. The line figure was formed by connecting 14 dots located at different joints and extremities of a typical human body (see Fig. 1). Each dot of the line figure was 15 pixels in size with the full figure being 280 

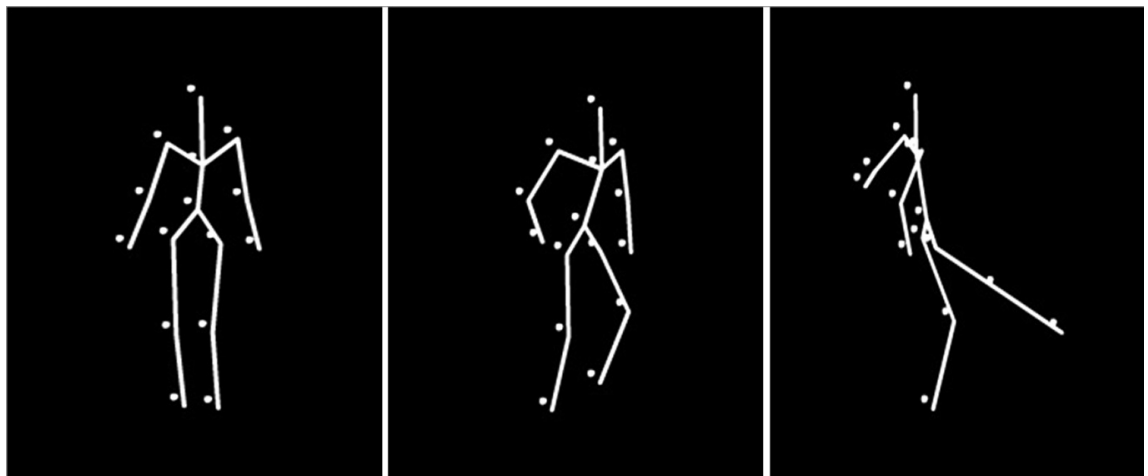

Figure 1. Still frames from three actions of the biological action identification task. The three actions are walking, climbing, and kicking (left to right).

pixels in size. The order of these trials was randomized on each participant. Participants were made aware of the 10 possible actions before the experiment, but were encouraged to respond intuitively. The 10 possible actions presented were catching, climbing stairs, jumping, jumping jacks, kicking, lifting, running, sitting, throwing, and walking. If participants, particularly children, did not have a word to describe the action performed by the BM figure, they were asked to imitate the action. On a number of occasions, participants who found it hard to give a verbal response for an action were able to recognize the action by imitating it, which was still taken as correct response. Participants reported the action verbally in Hindi, Telugu, or English, and the experimenter pressed the response button. Reaction times were not recorded.

The CC and DC group performed the CM and biological action identification task in a random order once. The normally SCs performed both tasks twice, with (VM control group) and without (SC group) the Bangerter filters; again, the order was randomized.

\section{Data analysis}

VA as measured using FrACT was recorded in logMAR units and the ability to detect CM was measured as the percentage of coherently moving dots needed to achieve $82 \%$ correct responses. The action task consisted of 10 actions presented from three different view angles. The number of correct responses obtained out of 30 was used to calculate the proportion of correct responses for each participant. VA (logMAR units), biological action identification (proportion of correct responses) and CM detection (threshold) were taken as dependent variables for separate group comparisons (CC, DC, VM/SC). One-way ANOVA was used to compare the CC group with the DC and VM group. A separate analysis was run comparing the CC group with the DC and the SC group. Planned comparisons between SC and VM groups were run using paired $t$ test. Homogeneity of data were tested using Levene's test, and when violated, the ANOVA was conducted assuming unequal variance.

The effect of viewpoint on the proportion of correct responses was additionally added as a repeated measurement factor in a two-way ANOVA with CC versus VM as group factor. The number of correct responses was averaged for all the 10 trials from a given viewpoint and the average score was taken as the proportion of correct responses for this view angle. All analyses were done with RStudio (RStudio Team, 2016) using appropriate packages. An effect was considered significant if the resulting $p$ value for the statistical test was $<0.05$.

\section{Result}

\section{VA comparison between groups}

A one-way ANOVA comparing the mean VA between the CC, DC, and VM groups was not significant $\left(F_{(2,33)}=1.11 ; p=0.34\right)$. However, the comparison of the mean VA between $\mathrm{CC}, \mathrm{DC}$, and SC groups using a oneway ANOVA was found to be significant $\left(F_{(2,16.45)}=41.2\right.$; $p<0.001)$. Post hoc pairwise $t$ tests using Bonferroni corrections showed that the VA score for the SC group (mean logMAR VA $=-0.13, \mathrm{SD}=0.11$ ) was significantly higher than the VA scores of both the CC (mean logMAR $\mathrm{VA}=0.753, \mathrm{SD}=0.33 ; p<0.001$ ) and the $\mathrm{DC}$ (mean $\log M A R$ VA $=0.556, S D=0.63 ; p=0.001$ ) group. Further,

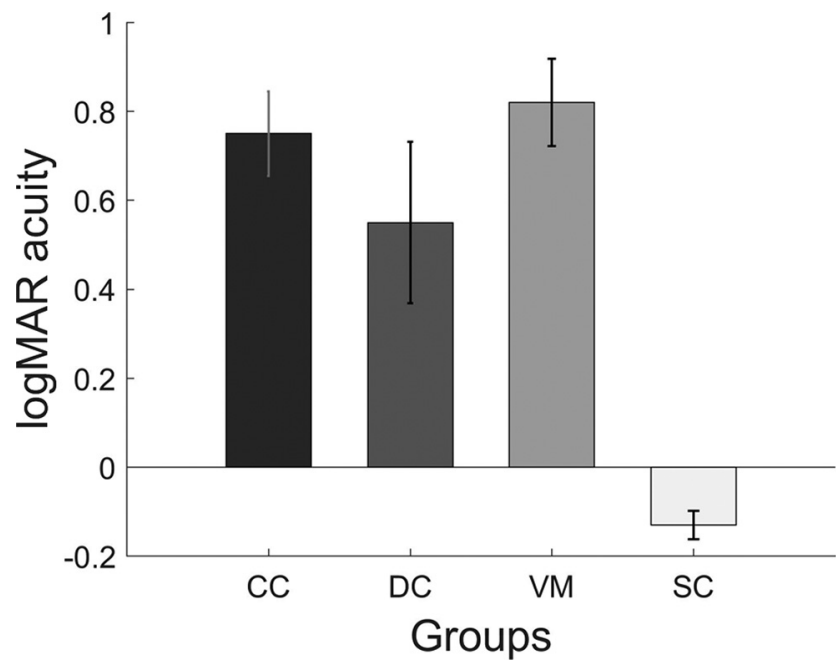

Figure 2. Bar plots displaying the visual acuities of each of group. Shown are the mean VA and the error bars indicate the SEM. 

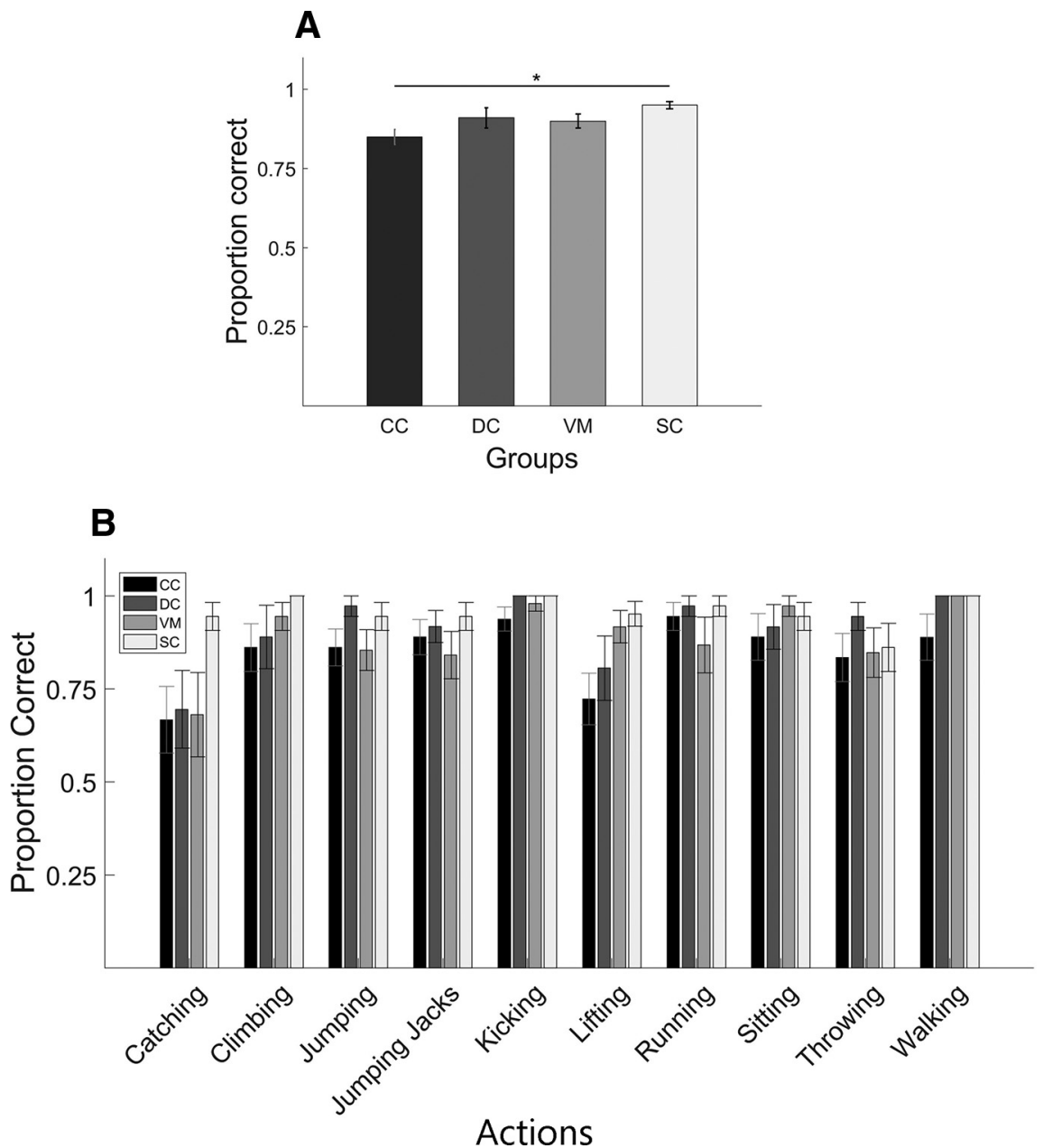

Figure 3. Results of the biological action identification task. $\boldsymbol{A}$, Bar plots display the proportion of correct responses for each group: CC, DC, VM, SC (for definition, see Fig. 1). Asterisk indicates significant group difference. $\boldsymbol{B}$, Same as $\boldsymbol{A}$ but proportion correct responses separately averaged for each of the 10 actions. Shown are group mean scores; error bars indicate SEM.

a within group comparison using paired $t$ test demonstrated the successful match of the Bangerter filters: The SC group had significantly better VA $\left(t_{(11)}=-10.13 ; p<\right.$ 0.001 ), than the $\mathrm{VM}$ group (mean logMAR $\mathrm{VA}=0.82$, $\mathrm{SD}=0.34$; Fig. 2).

\section{Biological action identification task: comparison between groups}

The one-way ANOVA with participant groups CC, DC, VM as between groups factor and proportion of correct responses as dependent variable found no significant effect of group $\left(F_{(2,33)}=1.44 ; p=0.24\right)$, whereas the analogous ANOVA with the participant groups CC, DC, and SC groups was found to be significant $\left(F_{(2,33)}=4.34 ; p=\right.$ $0.02)$. Post hoc pairwise $t$ tests using Bonferroni corrections for the latter showed that the CC group (mean score $=0.85, \mathrm{SD}=0.08)$ performed significantly worse in the task than the SC group (mean score $=0.95, \mathrm{SD}=0.04$; $p=0.019$ ), but the performance of CC group was not significantly different from the DC group (mean score $=0.91$, $\mathrm{SD}=0.11 ; p=0.25$; Fig. $3 A$ ). As seen in Figure $3 B$, performance varied across action type but the CC group

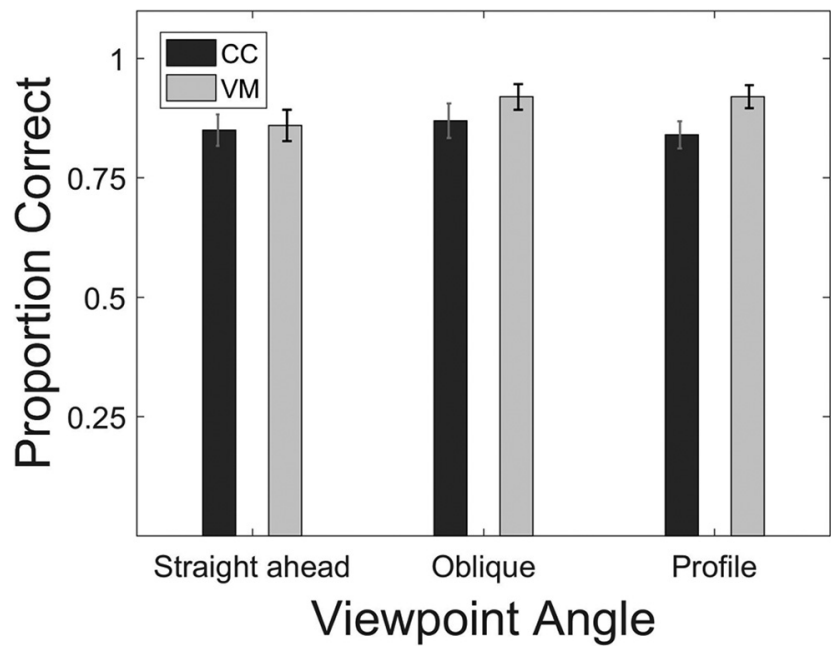

Figure 4. Biological action identification task. Bar plots show the proportion of correct responses separately averaged for the three viewpoints and CC and the VM participants (for definitions, see Fig. 1). Error bars indicate SEM. 

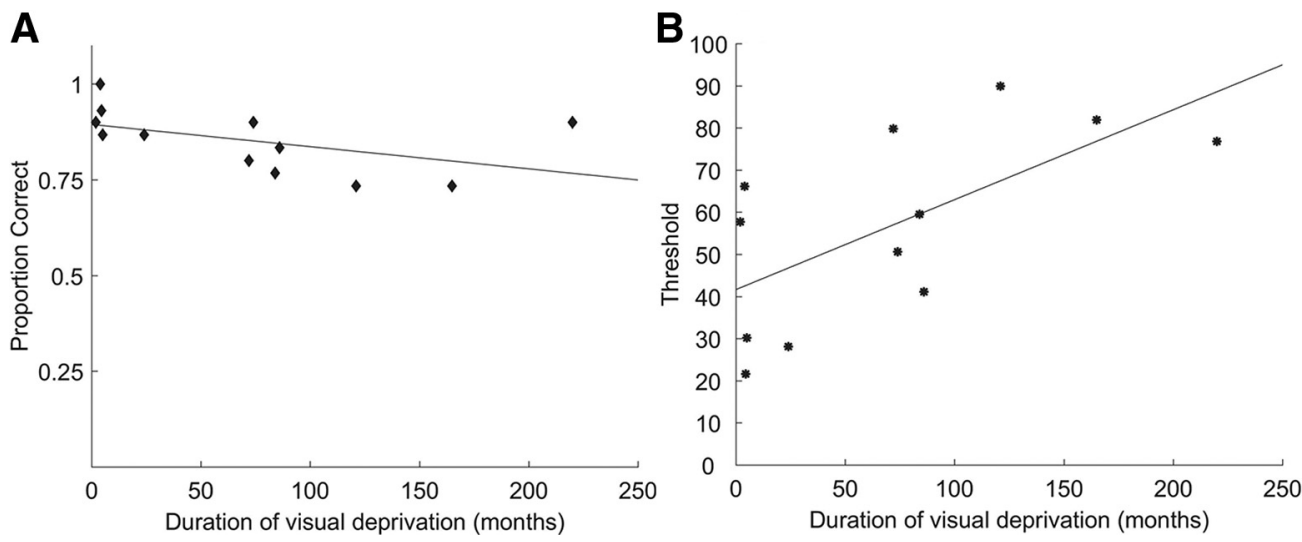

Figure 5. Scatter plot showing the performance of CC group in the $(\boldsymbol{A})$ biological action identification task and $(\boldsymbol{B})$ coherence motion detection task as a function of the duration of visual deprivation. Data points indicate single subjects. The data were fitted with a linear regression line (for definitions, see Fig. 1).

achieved a mean accuracy of $66.7 \%(S D=0.3)$ for the action they were least able to identify.

\section{Effect of viewpoint on biological action identification}

A mixed ANOVA with the between subject factor group (CC, VM) and the repeated measures factor viewpoint (straight, oblique, profile viewpoints) was run to test for the effect of viewpoint on action identification. There was neither a main effect of group $\left(F_{(1,22)}=1.577, p=0.22\right.$; pooled means: $C C=0.85$, $\mathrm{SD}=0.08 ; \mathrm{VM}=0.89, \mathrm{SD}=0.08)$ nor a main effect of viewpoint $\left(F_{(2,44)}=1.18, p=0.317\right.$, pooled means: straight $\quad$ ahead $=0.85, \quad \mathrm{SD}=0.11 ; \quad$ oblique $=0.89$, $S D=0.11$; profile $=0.87, S D=0.09$ ). Further, the interaction of group and viewpoint was non-significant $\left(F_{(2,44)}=0.942, p=0.397\right.$; Fig. 4).

\section{Effect of duration of visual deprivation on task performance}

A partial correlation between the duration of visual deprivation and the performance in biological action identification task after controlling for the duration of sight recovery following cataract surgery was not significant (Pearson's $r_{(10)}=-0.43 ; p=0.18 ;$ Fig. $5 A$ ). In contrast, the corresponding partial correlation for the $\mathrm{CM}$ task was significant (Pearson's $r_{(10)}=0.63, p=0.03$; Fig. $5 B$ ).

\section{Effect of VA on CM detection thresholds}

Detection thresholds for CM were compared between CC, DC, VM groups using a one-way ANOVA (Fig. 4). The group difference was found to be significant $\left(F_{(2,19.1)}=8.87 ; p=0.001\right)$. Post hoc group comparisons using Bonferroni corrected $t$ tests showed that the CM threshold for the CC group (mean threshold=56.9, $S D=22.9)$ was significantly higher than both for the $D C$ (mean threshold $=33.9, \mathrm{SD}=25.4 ; p=0.03$ ) and the VM group (mean threshold $=25.5, \mathrm{SD}=11.1 ; p=0.002$ ), while the DC and the VM group did not differ $(p=0.98)$. In a separate analysis, the one-way ANOVA comparing the CC, DC, and SC groups revealed a significant group effect
$\left(F_{(2,33)}=7.95 ; p=0.001\right)$. Bonferroni corrected group comparisons found significantly higher $\mathrm{CM}$ thresholds for the CC group than for the SC group (mean threshold $=20.9$, $\mathrm{SD}=18.2 ; p=0.001)$. Again, the DC group did not significantly differ from the SC group $(p=0.49)$. Blurring the visual stimuli did not significantly reduce $\mathrm{CM}$ thresholds in the normally SC group $\left(t_{(11)}=0.98, p=0.34\right)$ as tested using a paired $t$ test comparing the VM and SC groups (Fig. 6).

\section{Discussion}

BM detection thresholds in sight recovery individuals with a history of congenital cataract have been found to be indistinguishable compared with both typical SCs and individuals with a history of DC (Hadad et al., 2012; Bottari et al., 2015). In the present study, we tested a

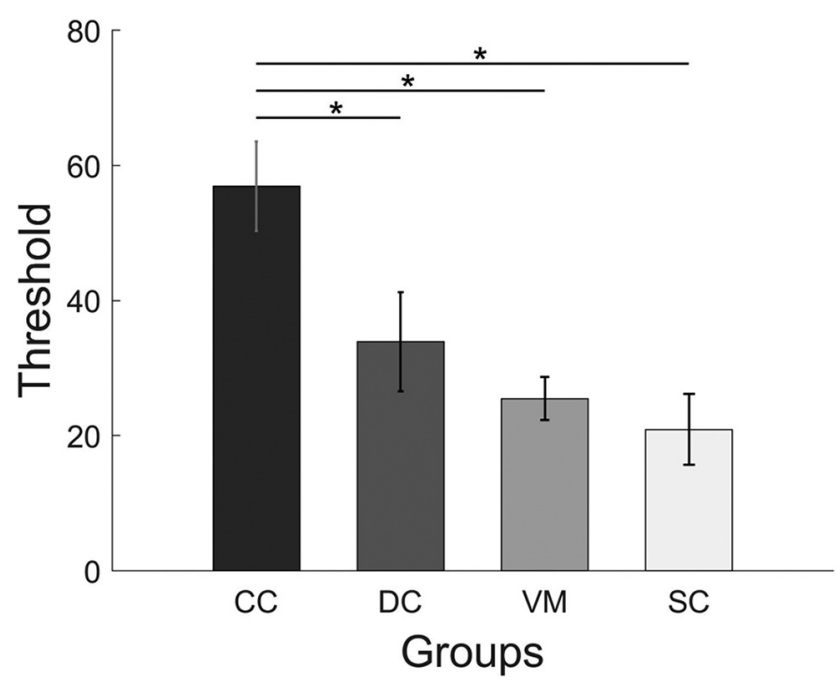

Figure 6. $\mathrm{CM}$ thresholds. Bars display $\mathrm{CM}$ thresholds in each group; CC, DC, VM, SC (for definitions, see Fig. 1). The $y$-axis specifies the percentage of dots moving coherently to detect the $\mathrm{CM}$ with an accuracy of $82 \%$. Shown are mean scores, and the error bars indicate SEM. Asterisks indicate significant group differences. 
group of individuals with CC (CC group) after cataract removal surgery (a few of whom had had long lasting visual deprivation of up to $\sim 18$ years) in a more complex BM task, that is, the identification of human actions. Three control groups were employed: to test for unspecific effects of cataract surgery and for the importance of visual pattern input at birth, we ran a group of DC reversal individuals (DC group); to test for the effects of persisting VA loss, we included normally SCs both with (VM group) and without (SC group) a blurring of their sight. Additionally, a $\mathrm{CM}$ task was performed by the same individuals. Despite some CC individuals still suffering severe acuity loss after cataract removal surgery they were able to recognize human actions with an impressive precision (mean accuracy of $85 \%$ ). The VM group's results demonstrated that the slightly but statistically significant lower performance of the CC compared with the SC group could be accounted for by overall lower VAs at the time of testing. This finding for action identification was in stark contrast to the results in the $\mathrm{CM}$ task: $\mathrm{CC}$ individuals performed worse than all three control groups. This pattern of results in the same individuals provides strong evidence for an impressive resilience of complex BM processing, such as recognizing human actions to aberrant visual experience after birth.

Detecting BM has been shown to be present in human infants (Bertenthal et al., 1987; Simion et al., 2008) and other animals such as chicks (Vallortigara et al., 2005). BM processing does not seem to rely on typical temporal motion processing areas, since lesions in these areas do not affect participants' ability to perceive BM (Vaina et al., 1990), and their ability to identify actions performed by human point light displays (McLeod, 1996). Here we tested specific actions, almost exclusive of human beings such as bipedal climbing stairs and kicking. Neurons responding to BM and actions have been observed in a number of brain areas such as F5 of the premotor cortex in macaque monkeys (Giese and Poggio, 2003). In macaques, neurons of the superior temporal sulcus (STS) have been shown to respond to full body movement (Oram and Perrett, 1994), and these neurons have been found to be multisensory (Bruce et al., 1981). The action-related (Grossman et al., 2004) and multisensory nature (BidetCaulet et al., 2005; Nath and Beauchamp, 2011) of the STS have been demonstrated in humans too. Hence, it could be speculated that during development, these neurons acquire their functional tuning by means of auditory and proprioceptive cues of self-motion. After sight restoration, CC individuals might associate the cues of selfmotion from the multisensory neurons with the newly available visual cues, which in turn allows them to learn to recognize visual actions. This idea is compatible with brain imaging studies in congenitally blind humans which observed an activation of premotor and parieto-temporal areas to sounds produced by human actions; the activation overlapped with that found in sighted people for both auditory and visual actions (Ricciardi et al., 2009). Alternatively, it could be argued that the recognition of visual actions is an innate ability of humans possibly because of its high relevance for survival. This idea is expressed in the concept of perceptual life detectors (Troje and Westhoff, 2006). The finding that newborns as young as $2 \mathrm{~d}$ preferentially look at biological movement (Simion et al., 2008) is compatible with this idea. However, detecting BM does not mean that infants were able to recognize actions. In particular, actions which involve some artefacts, such as kicking or catching a ball seem to be unlikely innate. A third alternative is that the ability to identify human actions was predominantly visually learned after sight restoration. We did not find a significant correlation between duration of deprivation and performance in the biological action identification task after taking the duration since cataract removal into account (partial correlation Pearson's $r_{(10)}=-0.43 ; p=$ $0.18)$. Although we hesitate to interpret a null finding in a relatively small sample size, this finding is at least compatible with the idea that the exact time of vision restoration does not matter for learning to recognize human actions as would be predicted by the existence of a sensitive period. Humans might thus be predisposed to learn biological actions. It might be argued that remaining pre-surgery vision had allowed CC individuals to acquire knowledge of typical BM patterns for human actions. However, most CC participants did not have useful pre-surgery vision and the one with useful vision had absorbed lenses (suggesting that the lenses had been dense at birth). Moreover, this participant (CC12) and a second participant with more than light sensation before surgery (CC4) belonged to the worst performing CC individuals $(76 \%$ and $73 \%$ correct, respectively), likely because of their relatively low postsurgery VA (see Table 1).

Another astonishing finding of the present study was the ability of $\mathrm{CC}$ individuals to recognize human actions independent of the viewpoint similarly as found for the control groups. This is a remarkable finding, since it has been reported that CC individuals have impairments in recognizing faces from different viewpoints (Putzar et al., 2010; de Heering and Maurer, 2014). In fact, the latter causes a severe impairment in $\mathrm{CC}$ individuals in recognizing people by their face in everyday situations. Neuroscience studies have provided evidence that neural systems typically specialized for face processing, including the extraction of face invariant features, lack the functional specificity in CC individuals (Röder et al., 2013; Grady et al., 2014). Since face processing improves with functional differentiation of the fusiform gyrus, a core area of the face processing system (Cantlon et al., 2011), it was speculated that a non-specialized neural system allows for simple functions like face detection and matching but not for the extraction of face invariant features which would allow for the face identity recognition in different contexts. Interestingly, Bottari et al. (2015) demonstrated in the same CC individuals who did not show a face specific neurophysiological response, a selective event-related potential response for intact versus scrambled BM, suggesting that the neural systems of BM processing acquired a functional specialization despite early visual deprivation. These lines of evidence suggest that the neural system for face and BM processing can be dissociated. In fact, a recent study in patients with lesions in the ventral temporal cortex 
including the fusiform gyrus found that they had intact BM perception (Gilaie-Dotan et al., 2015) including action identification despite their severe impairments in face processing. Further, controlling for VA, the present study suggests that low vision somewhat attenuates action identification but that action identification is still high even under low VA conditions.

In contrast to BM processing, CM processing was impaired in the CC group. In accordance with previous reports (Burton et al., 2015), we found that CM processing threshold did not decrease by blurring the stimuli in the VM group. Thus, the deficit observed in CC individuals cannot be explained by trivial VA differences. Moreover, $\mathrm{CM}$ thresholds varied with the age at surgery in the CC individuals: the longer their visual deprivation had lasted, the worse their performance in the CM task. In the context of indistinguishable $\mathrm{CM}$ thresholds in the SC and VM group, the present results suggest that the CM processing deficits of the $\mathrm{CC}$ individuals must be because of changes in the neural circuits associated with $\mathrm{CM}$ processing. In fact, a previous EEG study reported that $\alpha$ oscillations which have been associated with global motion processing (Händel et al., 2007) were greatly reduced in CC individuals (Bottari et al., 2016). It could be argued that the selective deficit in CM perception of CC individuals was predominantly because of the presence of nystagmus in this group. Such deficits for nystagmus patients (with other etiologies than congenital cataract) have mostly been found for slower motion velocities than used in the present study (Shallo-Hoffmann et al., 1998; Neveu et al., 2009). One study (but see Shallo-Hoffmann et al., 1998) observed deficits particular for vertical motion velocity discrimination, that is, motion perpendicular to the direction of the nystagmus (Neveu et al., 2009). However, vertical motion processing deficits would predict a particular impairment of CC individuals for BM tasks as used in the present and in a previous study (Bottari et al., 2016) which unlike the CM task comprised vertical motion components. However, this is not what was found in the present study. Further, despite the presence of nystagmus in all participants CM thresholds considerably varied in the CC group. Therefore, while it is not possible to entirely exclude some effect of nystagmus on $\mathrm{CM}$ processing, it seems rather unlikely that the presence of nystagmus could explain the current pattern of results. Finally, given the relatively low number of dots used in the CM detection task, it could be claimed that the CC group did not use motion cues as effectively as the SC group. This is unlikely because the VM group used such cues similarly efficiently as the SC group. Additionally, to use a dot size compatible with the participants' VA, the number of dots in the CM display was much lower than in most previous studies investigating the motion coherence thresholds. This may have limited the lowest thresholds that could have been measured in the SC and VM groups. However, the higher thresholds of the CC group would not have been subject to this limitation, and so the difference of coherence thresholds between groups as observed in the present study, if affected, might be an underestimate.

It might be argued that the performance in the biological action identification task was at ceiling and thus the task was much easier than the CM task resulting in the simple dissociation observed in the present study. Of course, it is not possible to compare the outcome of the two tasks on the same scale. However, we consider this account for the pattern of results in the present study as unlikely: First, the biological action identification task, unlike the $\mathrm{CM}$ task worsened by blurring. Moreover, some items were more difficult than others (e.g., "catching" was the most difficult) demonstrating that performance was not at ceiling. Interestingly, the relative ease or difficulty of action identification for each item was consistent across groups, suggesting the involvement of similar processing mechanisms. Second, the high performance in the biological action identification task of CC individuals was in accordance with indistinguishable thresholds to detect BM (Hadad et al., 2012; Bottari et al., 2015). Third, global motion processing has been shown to be impaired in a number of developmental disorders such as fragile $\mathrm{X}$, preterm infants, dyslexia, amblyopia and hemiplegia (for review, see Braddick et al., 2017), and these findings have led to the suggestion that dorsal stream functions are highly vulnerable during development (Braddick et al., 2003). The present results of the CC group are in line with this idea. Vulnerability to visual deprivation is the downside of high experience dependence and plasticity. Therefore, we would expect that individuals who particularly rely on visual motion processing from early on, such as congenitally deaf individuals, make use of this enhanced plasticity to acquire an extraordinarily high ability in visual motion processing. In fact, lower thresholds for global motion detection (Neville and Lawson, 1987) and enhanced neural processing (Hauthal et al., 2013; Retter et al., 2019) have been observed in deaf humans (Neville et al., 1983). As suggested by Stevens and Neville (2009), sensitive periods are a double edged sword: the lack of experience results in permanently impaired functioning and a particularly challenging stimulation results in enhanced functioning.

In sum, we demonstrate an impressive sparing of a complex visual function, that is, the identification of human actions in a group of sight recovery individuals who had suffered a congenital loss of pattern vision for up to 18 years. By controlling for VA as well as for unspecific effects of cataract surgery and the onset of visual deprivation, we furthermore provide in the same individuals strong additional evidence for an experience dependent development of CM processing during a sensitive period in early ontogeny.

\section{References}

Agervi P (2011) Treatment with Bangerter filters. J AAPOS 15:121122. 
Bach M (1996) The Freiburg Visual Acuity Test-Automatic measurement of visual acuity. Optom Vis Sci 73:49-53.

Bertenthal BI, Proffitt DR, Kramer SJ (1987) Perception of biomechanical motions by infants: implementation of various processing constraints. J Exp Psychol Hum Percept Perform 13:577-585.

Bidet-Caulet A, Voisin J, Bertrand O, Fonlupt P (2005) Listening to a walking human activates the temporal biological motion area. Neuroimage 28:132-139.

Bottari D, Troje NF, Ley P, Hense M, Kekunnaya R, Röder B (2015) The neural development of the biological motion processing system does not rely on early visual input. Cortex 71:359-367.

Bottari D, Troje NF, Ley P, Hense M, Kekunnaya R, Röder B (2016) Sight restoration after congenital blindness does not reinstate alpha oscillatory activity in humans. Sci Rep 6:24683.

Bottari D, Kekunnaya R, Hense M, Troje NF, Sourav S, Röder B (2018) Motion processing after sight restoration: no competition between visual recovery and auditory compensation. Neuroimage 167:284-296.

Braddick O, Atkinson J, Wattam-Bell J (2003) Normal and anomalous development of visual motion processing: motion coherence and "dorsal-stream" vulnerability. Neuropsychologia 41:1769-1784.

Braddick O, Atkinson J, Akshoomoff N, Newman E, Curley LB, Gonzalez MR, Brown T, Dale A, Jernigan T (2017) Individual differences in children's global motion sensitivity correlate with TBSSbased measures of the superior longitudinal fasciculus. Vision Res 141:145-156.

Brenner E, Cornelissen F, Nuboer W (1990) Striking absence of longlasting effects of early color deprivation on monkey vision. Dev Psychobiol 23:441-448.

Bruce C, Desimone R, Gross CG (1981) Visual properties of neurons in a polysensory area in superior temporal sulcus of the macaque. J Neurophysiol 46:369-384.

Burton EA, Wattam-Bell J, Rubin GS, Atkinson J, Braddick O, Nardini M (2015) The effect of blur on cortical responses to global form and motion. J Vis 15:1-14.

Cantlon JF, Pinel P, Dehaene S, Pelphrey KA (2011) Cortical representations of symbols, objects, and faces are pruned back during early childhood. Cereb Cortex 21:191-199.

Clifford CWG, Webster MA, Stanley GB, Stocker AA, Kohn A, Sharpee TO, Schwartz O (2007) Visual adaptation: neural, psychological and computational aspects. Vision Res 47:3125-3131.

de Heering A, Maurer D (2014) Face memory deficits in patients deprived of early visual input by bilateral congenital cataracts. Dev Psychobiol 56:96-108.

Ellemberg D, Lewis TL, Maurer D, Lui CH, Brent HP (1999) Spatial and temporal vision in patients treated for bilateral congenital cataracts. Vision Res 39:3480-3489.

Ellemberg D, Lewis TL, Maurer D, Brar S, Brent HP (2002) Better perception of global motion after monocular than after binocular deprivation. Vision Res 42:169-179.

Gelbart SS, Hoyt CS, Jastrebski G, Marg E (1982) Long-term visual results in bilateral congenital cataracts. Am J Ophthalmol 93:615621.

Giese MA, Poggio T (2003) Neural mechanisms for the recognition of biological movements. Nat Rev Neurosci 4:179-192.

Gilaie-Dotan S, Saygin AP, Lorenzi LJ, Rees G, Behrmann M (2015) Ventral aspect of the visual form pathway is not critical for the perception of biological motion. Proc Natl Acad Sci USA 112:E361E370.

Grady CL, Mondloch CJ, Lewis TL, Maurer D (2014) Early visual deprivation from congenital cataracts disrupts activity and functional connectivity in the face network. Neuropsychologia 57:122-139.

Grossman ED, Blake R, Kim C-Y (2004) Learning to see biological motion: brain activity parallels behavior. J Cogn Neurosci 16:1669-1679.

Hadad B, Maurer D, Lewis TL (2012) Sparing of sensitivity to biological motion but not of global motion after early visual deprivation. Dev Sci 15:474-481.
Händel B, Lutzenberger W, Thier P, Haarmeier T (2007) Opposite dependencies on visual motion coherence in human area $\mathrm{MT}+$ and early visual cortex. Cereb Cortex 17:1542-1549.

Hauthal N, Sandmann P, Debener S, Thorne JD (2013) Visual movement perception in deaf and hearing individuals. Adv Cogn Psychol 9:53-61.

Johansson G (1973) Visual perception of biological motion and a model for its analysis. Percept Psychophys 14:201-211.

Le Grand R, Mondloch CJ, Maurer D, Brent HP (2001) Early visual experience and face processing. Nature 410:890.

Lewis TL, Maurer D (2009) Effects of early pattern deprivation on visual development. Optom Vis Sci 86:640-646.

McKyton A, Ben-Zion I, Doron R, Zohary E (2015) The limits of shape recognition following late emergence from blindness. Curr Biol 25:2373-2378.

McLeod P (1996) Preserved and impaired detection of structure from motion by a "motion-blind" patient. Vis Cogn 3:363-392.

Nath AR, Beauchamp MS (2011) Dynamic changes in superior temporal sulcus connectivity during perception of noisy audiovisual speech. J Neurosci 31:1704-1714.

Neveu MM, Jeffery G, Moore AT, Dakin SC (2009) Deficits in local and global motion perception arising from abnormal eye movements. J Vis 9:9-15.

Neville HJ, Lawson D (1987) Attention to central and peripheral visual space in a movement detection task: an event-related potential and behavioral study. II. Congenitally deaf adults. Brain Res 405:268-283.

Neville HJ, Schmidt A, Kutas M (1983) Altered visual-evoked potentials in congenitally deaf adults. Brain Res 266:127-132.

Newsome W, Paré E (1988) A selective impairment of motion perception following lesions of the middle temporal visual area (MT). J Neurosci 8:2201-2211.

Odell NV, Leske DA, Hatt SR, Adams WE, Holmes JM (2008) The effect of Bangerter filters on optotype acuity, vernier acuity, and contrast sensitivity. J AAPOS 12:555-559.

Oram MW, Perrett DI (1994) Responses of anterior superior temporal polysensory (STPa) neurons to "biological motion" stimuli. J Cogn Neurosci 6:99-116.

Pérez GM, Archer SM, Artal P (2010) Optical characterization of bangerter foils. Invest Ophthalmol Vis Sci 51:609-613.

Pitchaimuthu K, Sourav S, Bottari D, Banerjee S, Shareef I, Kekunnaya R, Röder B (2019) Color vision in sight recovery individuals. Restor Neurol Neurosci 37:583-590.

Putzar L, Hötting K, Rösler F, Röder B (2007) The development of visual feature binding processes after visual deprivation in early infancy. Vision Res 47:2616-2626.

Putzar L, Hötting K, Röder B (2010) Early visual deprivation affects the development of face recognition and of audio-visual speech perception. Restor Neurol Neurosci 28:251-257.

Retter TL, Webster MA, Jiang F (2019) Directional visual motion is represented in the auditory and association cortices of early deaf individuals. J Cogn Neurosci 31:1126-1140.

Ricciardi E, Bonino D, Sani L, Vecchi T, Guazzelli M, Haxby JV, Fadiga L, Pietrini P (2009) Do we really need vision? How blind people "see" the actions of others. J Neurosci 29:9719-9724.

Röder B, Ley P, Shenoy B, Kekunnaya R, Bottari D (2013) Sensitive periods for the functional specialization of the neural system for human face processing. Proc Natl Acad Sci USA 110:1676016765.

Rogers GL, Tishler CL, Tsou BH, Hertle RW, Fellows RR (1981) Visual acuities in infants with congenital cataracts operated on prior to 6 months of age. Arch Ophthalmol 99:999-1003.

RStudio Team (2016) RStudio: integrated development for $R$. Boston: RStudio, Inc.

Rutstein RP, Foster NC, Cotter SA, Kraker RT, Lee DH, Melia M, Quinn GE, Tamkins SM, Wallace DK, Pediatric Eye Disease Investigator Group (2011) Visual acuity through Bangerter filters in nonamblyopic eyes. J AAPOS 15:131-134.

Saunders R, Troje N (2011) A test battery for assessing biological motion perception. J Vis 11:686-686. 
Shallo-Hoffmann JA, Bronstein AM, Acheson J, Morland AB, Gresty MA (1998) Vertical and horizontal motion perception in congenital nystagmus. Neuroophthalmology 19:171-183.

Simion F, Regolin L, Bulf H (2008) A predisposition for biological motion in the newborn baby. Proc Natl Acad Sci USA 105:809-813.

Stevens C, Neville H (2009) Profiles of development and plasticity in human neurocognition. In: The new cognitive neurosciences (Gazzaniga MS, ed), pp 165-181. Cambridge: The MIT Press.

Theusner S, de Lussanet M, Lappe M (2014) Action recognition by motion detection in posture space. J Neurosci 34:909-921.

Troje NF, Westhoff C (2006) The inversion effect in biological motion perception: evidence for a "life detector"? Curr Biol 16:821824.
Tytla ME, Lewis TL, Maurer D, Brent HP (1993) Stereopsis after congenital cataract. Invest Ophthalmol Vis Sci 34:17671773.

Vaina LM, Lemay M, Bienfang DC, Choi AY, Nakayama K (1990) Intact "biological motion" and "structure from motion" perceptionin a patient with impaired motion mechanisms: a case study. Vis Neurosci 5:353-369.

Vallortigara G, Regolin L, Marconato F (2005) Visually inexperienced chicks exhibit spontaneous preference for biological motion patterns. PLoS Biol 3:e208.

Webster MA (2015) Visual adaptation. Annu Rev Vis Sci 1:547-567.

Wiesel TN, Hubel DH (1965) Extent of recovery from the effects of visual deprivation in kittens. J Neurophysiol 28:1060-1072. 